\title{
Reliability Evaluation and Analysis of Mobile Ad Hoc Networks
}

\author{
Deepak Kumar Panda ${ }^{1}$, Ranjan Kumar Dash', \\ ${ }^{1}$ SOA University, Bhubaneswar, India \\ ${ }^{2}$ College of Engineering and Technology, Bhubaneswar, India
}

\begin{tabular}{l}
\hline \hline Article Info \\
\hline Article history: \\
Received Aug 21, 2016 \\
Revised Oct 21, 2016 \\
Accepted Nov 5, 2016 \\
\hline
\end{tabular}

\section{Keyword:}

Mobility of mobile nodes

Reliability

Routing

Signal to noise ratio(SNR)

\begin{abstract}
The paper addresses the reliability problem of mobile ad hoc networks under link and node failure model. Node reliability is calculated as a function of no. of neighbor nodes, packet success rate, and device type and packet size. The presence of a link between any node pair is binary and its reliability is computed considering the distance between nodes and signal-to-noise ratio (SNR). An efficient algorithm is proposed to analyze and calculate the reliability of mobile ad hoc networks considering multiple routes from source and destination nodes. The effect of different parameters on node reliability and link reliability are analyzed and discussed. The network is simulated and analyzed using INET frame work. Reliability of two distinct cases of this simulation is evaluated. The simulated results and discussions ensure that evaluation of the reliability of any mobile ad hoc networks can be done easily and in an efficient manner by the proposed method.
\end{abstract}

Copyright (C) 2017 Institute of Advanced Engineering and Science. All rights reserved.

\section{Corresponding Author:}

Deepak Kumar Panda, SOA University, Bhubaneswar, India-751030.

Email: deepak_panda_2000@yahoo.com

\section{INTRODUCTION}

A mobile ad hoc network is consists of a set of mobile nodes operating in an infrastructure less network with a centralized administration. Communication is made by establishing wireless links among mobile nodes through their antennas. In a single-hop scenario, a mobile node is not be able to communicate directly with other node due to limited radio power utilization of channel. Under such situations, communication is made possible by a multi-hop scenario, in which the source node communicates to the sink node by sending packets that must be relayed by several intermediate hosts [1].

The MANET is a infrastructure less wireless network. These networks have no fixed routers. Every node could be act as a router. The responsibilities for organizing and controlling the network are handled by the nodes themselves. The whole network is mobile, and due to mobility of the nodes the individual terminals are allowed to move freely [2-4]. Mobile Ad-hoc Networks are used for disaster management, communications during war, catastrophic failure and rescue operations when the infrastructure based network is not available [5-7]. It can provide a possible means for communications and information contact.

Extensive works have been carried out in evaluating and analyzing of reliability of wired networks [8-10]. Nevertheless, the works carried out for evaluating reliability of MANET are very limited [11-18]. Chen \&Lyu [14] inspected the problem of two terminal reliability in wireless networks resembled by Common Object Request Broker Architectures (CORBA) specifications. Chen \& Lyu also suggested a new reliability term i.e. the end-to-end expected instantaneous reliability (EIR) for accommodating the hand-over processes and alternative communication structures in wireless CORBA specifications. However, they presumed that only nodes are vulnerable to failures whereas links are faultfree. Two algorithm were suggested by Abo El Fotoh et al. [15] i.e. one for computing the two-terminal reliability and the other for computing the probable and the maximum message delay between sensors and the data sink in an operational distributed sensor networks (DSN). In aforesaid work, failures of stationary 
nodes are addressed while assuming links to be fault-free. Both works [14] and [15] discounted links failures and the nodes under study are either static or their movement is comprehended by the hand-off process. In reality, the reliability aspects of mobile and wireless networks infer that each node in a wireless network is always vulnerable to failure and the reliability of the overall network depends on the constituents' reliability and the degree of redundancy in the wireless network design [16]. Cook et al [17] Computes the two-terminal reliability of mobile ad hoc networks considering the mobility of the node and constant changes in network's connection. But, node reliability has not been addressed in their method. Further, the capacity of every link was assumed to be binary and homogeneous. The method presented in [18] is an extended work of [17] considering the node reliability. But, certain factors affecting link reliability e.g. distance, signal-to-noise ratio (snr) have not taken into consideration while calculating the link reliability. In [20] the researcher has nicely explained about scalability and quality of service of the nodes in MANET. In [21] the author has nicely explain about the inter node distance, routing of data packet in congested traffic in MANET.

From the study of literature carried out so far it is quite clear that although node and link of mobile ad hoc wireless networks are more prone to fail and there are many parameters responsible for such failures, still the problem has not been addressed in an efficient manner. Hence, it is utmost required to evaluate the reliability of such networks under node as well as link failure model considering certain parameters that may affect node reliability and link reliability. This motivates our study to propose new method to calculate and analyze node as well as link reliability considering the different parameters viz. distance, signal-to-noise ratio, no. of neighbor nodes, packet success rate, and packet size and device type. Further, a new algorithm is also proposed for the evaluation and analysis of the reliability of mobile ad hoc networks. The remaining of the paper is organized as follow: Section 2 proposes an efficient method for calculating node reliability and link reliability along with a new algorithm to analyze and evaluate the reliability of mobile ad hoc networks. Simulated results and discussion is presented in Section 3. Section 4 concludes the paper with its future scope.

\section{PROPOSED METHOD}

A mobile ad hoc network (MANET) can be modeled as a probabilistic graph $\mathrm{G}(\mathrm{N})$ consisting of mobile nodes $\mathrm{N}$, where each node has a reliability of ri. The two-way wireless link exist between any two nodes $\mathrm{i}$ and $\mathrm{j}$ is represented by $\mathrm{li}, \mathrm{j}, \mathrm{i}, \mathrm{j}=1, \ldots, \mathrm{N}$ and $\mathrm{i} \neq \mathrm{j}$. The existence of a link is binary and is defined as

$$
l_{i, j}=\left\{\begin{array}{c}
1 \text { if there exists a link between a pair of nodes } i \text { and } j \\
0 \text { other wise }
\end{array}\right.
$$

The following assumptions have been made as per [18] :

a. The source node and sink node are known.

b. Each node is of the similar type.

c. The range of wireless transmission for every node and every pair of node within the range of wireless communication are considered to be linked.

d. The movement of host is the random way point mobility model [5].

e. The fading factors for different links are Rayleigh random variables [19].

The reliability of nodes and links are calculated by the following manner:

\subsection{Calculation of Node Reliability}

A node is said to be reliable as long as it can forward the packets that it receives. Thus, the node reliability is affected by the following factor:

1. Type and capacity of the node $(\alpha)$

2. No. of neighbor nodes along with their distances

3. Packet size $(\beta)$

4. $\quad$ Packet success rate $(\gamma)$

Considering the above mentioned factors, the reliability of a node(i) of a MANET can be expressed

as:

$$
r_{i}=2\left(e^{2 \alpha\left(g_{i}-\beta\right)}+1\right)^{-1} \times \gamma
$$

where, giis the no. of neighbor nodes of node $i$. 


\subsection{Calculation of Link Reliability}

The link reliability $\left(\mathrm{p}_{\mathrm{i}, \mathrm{j}}\right)$ of a wireless network is defined by way of the probability of correct reception of signal at the receiver's end and is mainly depends on the following factors:

1. transmit power

2. inter distance between communicating nodes(d)

3. Signal-to-Noise Ratio (snr)

Thus, $\mathrm{p}_{\mathrm{i}, \mathrm{j}}$ can be defined as

$$
\left.p_{i, j}=e^{\left(-d^{k} / s n r\right.}\right)
$$

where, $\mathrm{k}$ is the propagation power loss exponent (its value is assumed to be 2 to 4 )

\subsection{Calculation of Reliability}

The reliability of a mobile ad hoc network can be defined as the probability that any two nodes of the network have an operative path connecting them.

The path in a mobile ad hoc network can be established through a multi-hop route which is a sequence of nodes through, which the information is relayed from a source node, s, to a destination node, d, i.e.:

$$
\text { Path }=<\mathrm{s}, \mathrm{h}_{1}, \mathrm{~h}_{2}, \mathrm{~h}_{3} \cdots \mathrm{h}_{\mathrm{i}} \cdots, \mathrm{d}>
$$

where,

$$
\begin{aligned}
& \mathrm{hi}=\text { intermediate nodes or hops } \\
& \mathrm{h}=\text { no. oh hops } \\
& \mathrm{R}=\prod_{\mathrm{i}=1}^{\mathrm{h}} \mathrm{r}_{\mathrm{i}} \mathrm{p}_{\mathrm{i}, \mathrm{j}} \\
& \left.=\prod_{\mathrm{i}=1}^{\mathrm{h}} 2\left(\mathrm{e}^{2 \alpha\left(\mathrm{g}_{\mathrm{i}}-\beta\right)}+1\right)^{-1} \times \gamma \times \mathrm{e}^{\left(-\mathrm{d}^{\mathrm{k}}(\mathrm{i}, \mathrm{i}+1) / \operatorname{snr}(\mathrm{i}, \mathrm{i}+1)\right.}\right)
\end{aligned}
$$

where, $\mathrm{d}(\mathrm{i}, \mathrm{i}+1)$ is the inter node gap between $\mathrm{i}$ and $\mathrm{i}+1$ nodes and $\operatorname{snr}(i, i+1)$ is the signal-to-ratio between $\mathrm{i}$ and $\mathrm{i}+1$ nodes

Equation 4 can be taken to evaluate reliability of a mobile ad hoc network when there exists a single path between $\mathrm{s}$ and $\mathrm{t}$. But, in reality numerous paths may occur between $\mathrm{s}$ and $\mathrm{t}$ and hence, for this case the following algorithm is proposed to analyze and evaluate reliability of a mobile ad hoc network.

\section{Algorithm to Evaluate Reliability of Wireless Networks}

Reliability_MAN $(s, t, k, \alpha, \beta, \gamma)$

Establish a route Path $=<s, h_{1}, h_{2}, h_{3} \cdots h_{i} \cdots, d>$ from source $(s)$ to destination $(t)$ node

$R=0$

$j=1 / * j$ is the no. of routes $* /$

While (true)

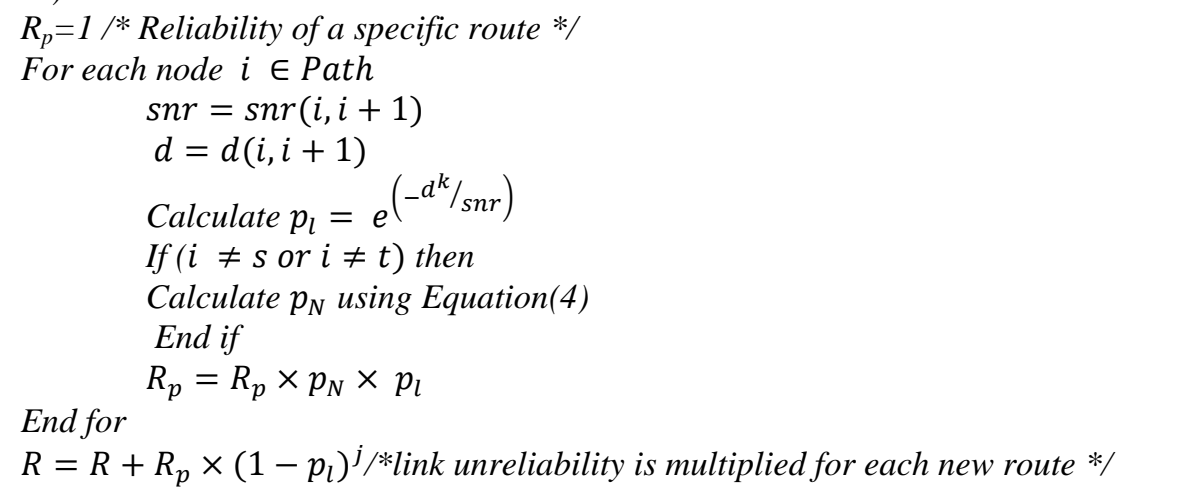


If $($ Route $=$ a new route be established from $s$ to $t)$ then

Path $=$ Route

$j=j+1$

Else

End if

Break

End while

Return R

\section{RESULTS AND DISCUSSIONS}

This section is separated into two parts: first part analyses the values of link reliability and node reliability with respect to different parameters such as inter node distance, SNR, number of neighbor nodes and the objective of this part is to study the behavior of reliability of links as well as nodes under several conditions. Further, the simulated results also validate the proposed method. The reliability of wireless networks is evaluated using the proposed method in the second part of this section.

For the purpose of simulation, the following parameters are set which is as per [18]: $\mathrm{k}=2, \alpha=1.0$, $\beta=10, \gamma=0.5, \mathrm{~d}=2, \mathrm{snr}=10$

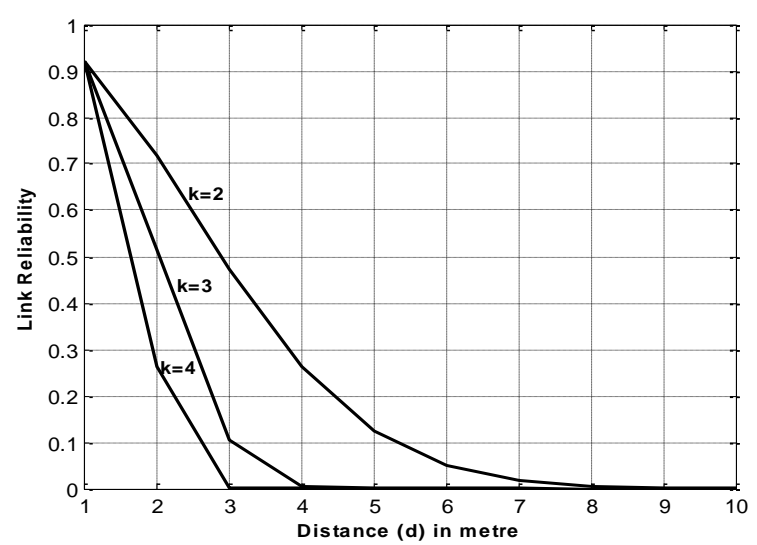

Figure 1. Effect of distance on Link Reliability

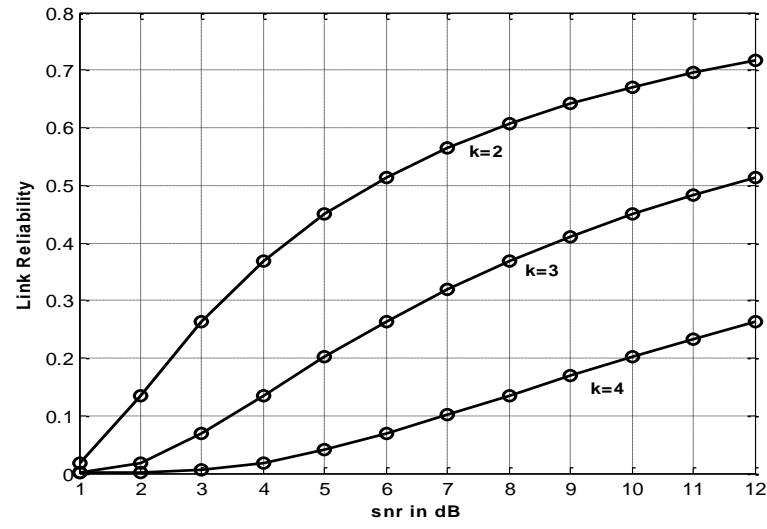

Figure 2. Effect of signal-to-noise ratio on Link Reliability

\subsection{Effect of Inter Node Distance on Link Reliability}

The link reliability decreases monotonically with respect to inter node distance due to fading (Figure 1). This is due the fact that with increase of distance, the attenuation goes on increasing which leads to reception of a poor signal at the receiver end. Additionally, the effect of the propagation power loss exponent $(\mathrm{k})$ on reliability is presented varying the value of $\mathrm{k}=2$ to 4 . Higher the value of this component lesser is the value of link reliability.

\subsection{Effect of snr on Link Reliability}

The values of link reliability is plotted against the values of snr ranging from 1 to 12 under different allowable values of propagation power loss exponent (Figure 2). From this figure, it can be observed that link reliability increases with respect to increasing the value of snr since it increases the signal strength.

\subsection{Effect of Neighbor Nodes on Node Reliability}

The different values of node reliability are calculated and plotted against the no. of neighbour nodes varying from 2 to 16 (Figure 3). The figure indicates high values of node reliability for up to 8 no. of neighbour nodes. For neighbour nodes of 9, 10, and 11, the computed reliability are $0.9,0.5$ and 0.1 respectively. The reliability is almost becomes 0 when no. of nodes reaches to 13 . Hence, each node can accommodate up to a maximum of 10 number of neighbour nodes. Such behaviour of reliability with respect to number of neighbour nodes is due to that fact that the increase of neighbours increases amount of packets arriving. When number of packet and packet arrival rate at a node increases to maximum as compared to 
receiving rate, it will overwhelm the receiver node. As a result congestion will occur and the rate of packet loss of the nodes will increase. Then it will leads to the unreliability of node.

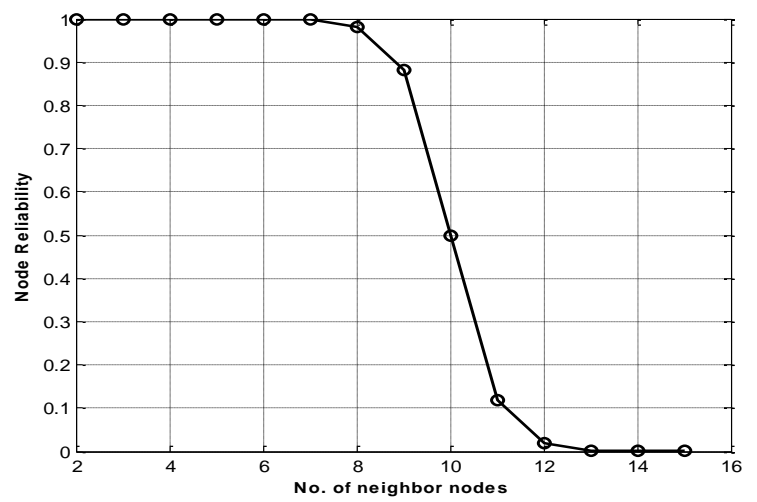

Figure 3. Effect of no. of neighbor nodes on node reliability

\subsection{Reliability Evaluation and Analysis of Mobile Ad Hoc Wireless Networks}

The terminal reliability of mobile ad hoc networks is evaluated, analyzed and simulated using INET framework varying the network size i.e. no. of nodes $(\mathrm{N})$ from 2 to 20 . The inter node distance (d) is set to $5 \mathrm{~m}$. The following two distinct cases of this simulation are discussed here:

1. Each node has N-1 no. of neighbor nodes.

2. Each node is just placed at a distance equal to $5 \mathrm{~m}$ from its neighbor node.

Case 1

The reliability evaluated using the proposed algorithm is plotted against the network size (Figure 4). From this figure, it is clear that the reliability of the network increases with respect to increase in network size up to 9 no. of nodes. This is due to fact of numerous paths (routes) exist between source and terminal nodes. The reliability value starts falling (when the network size is 10 no. of nodes) and even drops to 0 (when network size reaches to 14). This is due to more no. of neighbor nodes (which is discussed earlier in 3.4).

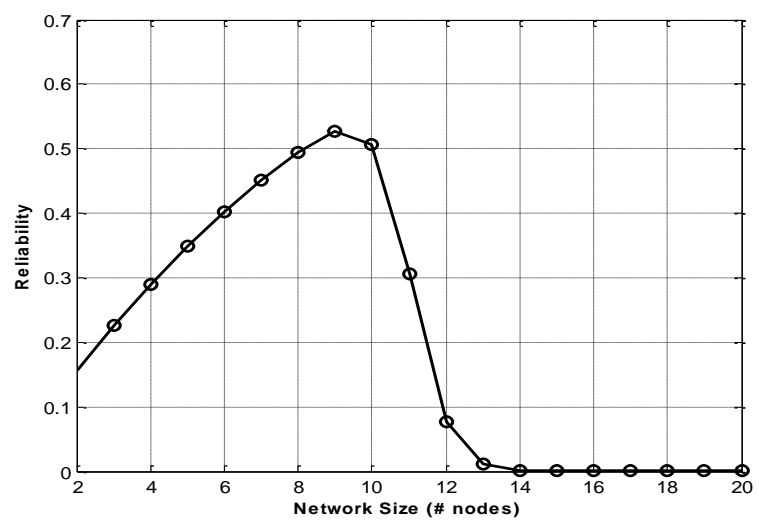

Figure 4. Reliability of mobile ad hoc networks of different sizes (Case-I)

\section{Case-II}

The computed reliability values for the different networks are plotted against the network size (Figure 5). The reliability value increases with respect to increase in network size as multiple paths can be established between $\mathrm{s}$ and $\mathrm{t}$. 


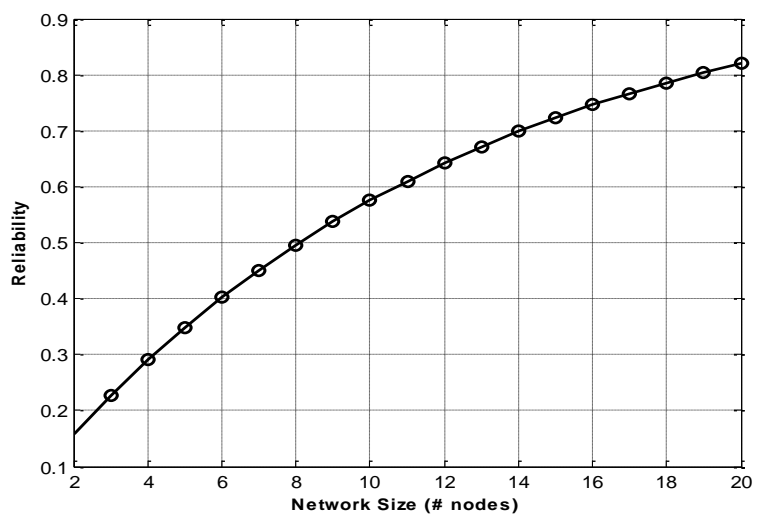

Figure 5. Reliability of mobile ad hoc networks of different sizes (Case-II)

\section{CONCLUSION}

The paper addresses both the node reliability and link reliability of mobile ad hoc networks. Node reliability is evaluated considering different parameters like no. of neighbor nodes, type and capacity of the node, packet success rate along with the data packet size. The link reliability is evaluated considering the inter node distances and signal-to-noise ratio (SNR). A new algorithm has been proposed to compute the reliability of mobile ad hoc wireless network. Effects of different parameters on node reliability and link reliability have been discussed. The network is simulated using INET framework. Reliability is evaluated and examined for two distinct cases of this simulation. From the simulated results and discussion, it is quite clear that the methods projected in this paper can be used to evaluate the reliability of mobile ad hoc networks under different failure models as well as under different conditions. The work carried out in this paper can be extended to find other measures of reliability such as broadcast reliability.

\section{REFERENCES}

[1] Pahlavan K, Krishnamurthy P. Principles of Wireless Networks. Englewood Cliffs: Prentice Hall PTR, (2002) 224229.

[2] Camp T, Boleng J, Davies V. A survey of mobility models for ad hoc network research wireless communication \& mobile computing (WCMC): special issue on mobile ad hoc networking: research. Trends, vol. 2, no. 5 (2002) 483-502.

[3] Zonoozi MM, Dassanayake P. User mobility modeling and characterization of mobility patterns. IEEE J Select Areas Commun, vol. 17, no. 7, (1997) 1239-52.

[4] Bhatt M, Chokshi R, Desai S, Panichpapiboon S, Wisitpongphan N, et al. Impact Of mobility on the performance of ad hoc wireless networks. IEEE 58th vehicular technology conference, vol. 5 (2003) 3025-9.

[5] Freebersyser JA, Leiner BA. DoD Perspective on mobile ad hoc networks. Ad Hoc Networking, (2001) 29-51.

[6] Leiner B, Ruth R and Sastry A.R.: Goals and challenges of the DARPA GloMo program, IEEE Personal Communications, vol. 3, no. 6, (1996) 34-43.

[7] Ruppe R., Griswald S., Walsh P., and Martin R. Near term digital radio (NTDR) system, Proceedings of IEEE MILCOM vol. 3, (1997), 1282-1287.

[8] Birolini Alessandro. Reliability Engineering: Theory and Practice. Springer; (2010).

[9] Dash RK, Tripathy CR. Polynomial Algorithms for Evaluation of Reliability of Parallel Computer Interconnection Systems, Iranian Journal of Electrical and Computer Engineering, vol. 9, no.1 (2010), 52-58.

[10] Mishra R and Chaturvedi S. A Cutsets-Based Unified Framework to Evaluate Network Reliability Measures, IEEE Transactions on Reliability, vol. 58, no. 4 (2009).

[11] Ye Z, Krishnamurthy SV, Tripathi SK. A routing framework for providing robustness to node failures in mobile ad hoc networks. Adhoc Networks, vol. 2, no. 1. (2004) 87-107.

[12] Luo J, Eugster PTh, Hubaux JP. Probabilistic reliable multicast in Ad hoc networks. Ad hoc Networks, vol. 2, no. $4(2004) 369-86$

[13] Abolhasan M, Wysocki T, Dutkiewicz E. A review of routing protocols for mobile ad hoc networks. Ad hoc Networks, vol.2, no. 1,(2004)1-22.

[14] Chen Z, Lyu MR. Reliability analysis for various communication schemes in wireless CORBA. IEEE Trans Reliab, vol. 54, no. 2, (2005)232-42.

[15] Abo EI Fotoh HM, Colbourn CJ. Computing 2-terminal reliability for radio-broadcast networks. IEEE Trans Reliab, vol. 38, no. 5(1989) 538-55.

[16] Andrew A.D.M., Snow P., Upkar Varshney. Reliability and Survivability of Wireless and Mobile Networks, Computer, vol. 33, no. 7, (2000) 49-55. 
[17] Cook J.L., Ramirez-Marque J.E. Two-terminal reliability analyses for a mobile ad hoc wireless network, Reliability Engg. \& System Safety, vol. 92 (2007) 821-829.

[18] Zhao X., You Z. and Wan H. A Novel Two Terminal Reliability Analysis for MANET, Journal of Applied Mathematics, (2013) 1-9.

[19] Khandani E., E. Modiano, J. Abounadi and L. Zheng, 2008. Reliability and route diversity in wireless networks. IEEE. Trans. Wireless Commun, vol. 7, (2008), 4772-4776.

[20] Pradeep Kumar K, B.R. Prasad Babu, "Investigating Open Issues in Swarm Intelligence for Mitigating Security Threats in MANET", International Journal of Electrical and Computer Engineering (IJECE), Vol. 5, No. 5, October 2015, pp. 1194 1201

[21] Ramanna Havinal, Girish V. Attimarad, M.N. Giri Prasad "EASR: Graph-based Framework for Energy Efficient Smart Routing in MANET using Availability Zones", International Journal of Electrical and Computer Engineering (IJECE), Vol. 5, No. 6, December 2015, pp. 1381 1395 